\title{
Yo ADORO A UNA RUBIA NORTEAMERICANA ${ }^{1}$
}

\author{
Carlos Gutiérrez Alfonzo
}

Resumen: En este texto se muestra cómo Santiago Serrano - poeta chiapaneco de la generación agrupada en torno a la compilación Fiesta de Pájaros, publicada por Héctor Eduardo Paniagua en 1932 - configura un tipo de mujer en una ciudad alejada de todas aquellas que habian aparecido hasta entonces en la poesía chiapaneca. Se analiza también cómo esa mujer fuera de lo común, con carácter, transforma al yo poético, quien habia vivido sumido en el sentimentalismo, en un ser dispuesto a perseguir una vida esforzada. Se pone el énfasis, por igual, en la necesidad de emprender una investigación hemerográfica que sitúe el poema en su justa dimensión, no sólo en el ámbito estatal, sino, sobre todo, en el de la poesía en lengua española.

Palabras clave: Poesía, anglicismo, paratextos, cuidad, mujer.

Carlos Gutiérrez Alfonzo, candidato a doctor en humanidades y artes, investigador del Centro de Estudios Superiores de México y Centroamérica de la UNICACH, cuerpo académico: Patrimonio sociocultural, especialidad: literatura y cultura, correo electrónico: carlosalfonzo6@hotmail.com.
Abstract: In this text it is shown how Santiago Serrano — poet chiapaneco of the generation contained around the compilation Party of Birds, published by Héctor Eduardo Paniagua in 1932 — configures woman's type in a city far from all those that had appeared until then in the poetry chiapaneca. It is also analyzed how that woman outside of the common thing, with character, transforms to the me poetic who had lived sunk in the sentimentality, in a being willing to pursue a made an effort life. It puts on the emphasis, equally, in the necessity of not only undertaking an investigation hemerographic that locates the poem in their fair dimension, in the state ambit, but, mainly, in that of the poetry in Spanish language.

Key words: Poetry, anglicism, paratextos, city, woman

\section{I}

$\mathrm{E}$ stá fechado en New York, en 1917, y es uno de los que Santiago Serrano entregó, con el título de "Yo Adoro a una Rubia...", 2 para su publicación en el libro promovido por Héctor Eduardo Paniagua, Fiesta de pájaros, editado en 1932, en Tuxtla Gutiérrez, Chiapas. He aquí el poema:

Yo adoro a una rubia norteamericana que juega lawn-tennis y baila fox-trot. La ví una mañana 
en New York,

bajo un sol de fuego corretear ufana

sobre la rudeza de un campo de sport.

\section{Me la presentaron}

y sus rudas manos mi mano estrujaron

y sus ojos glaucos me sugestionaron.

\section{Poeta}

de musa llorona

y romanticismo de Romeo y Julieta,

- me dijo la inquieta

amazona;- -

basta ya de sueños, todo humo y mentira; 15

antes que un Apolo serás un atleta.

Preciso es que trueques la lira

por una raqueta.

Y aquella amazona

que tiene maldad de sirena,

cortó mi melena

y ajó como quiso mi pobre persona.

En pocas semanas

curó mi idealismo

y con risas locas y muecas paganas

saturó mi vida de materialismo.

Por ella he triunfado.

Hoy media un abismo

entre mi presente vida de esforzado

y mi ya olvidado

sentimentalismo.

Está ya lejana

mi vida de sueño. ${ }^{3} \mathrm{Mi}$ amor

es ya de la rubia norteamericana

que juega lamn-tennis y baila fox-trot.

35

New York, 1917.

Santiago Serrano nació en 1896 (Morales Bermúdez, 1997: 150) —año en el cual Rubén Darío publicó Prosas profanas, en cuyas palabras preliminares el poeta nicaragüense dice que detesta la vida y el tiempo en el que le tocó nacer-. Se le identifica con el grupo que José Casahonda Castillo denominó la "generación de Chanti" — diminutivo de Santiago—, conformada por la mayoría de los poetas con los que se integró Fiesta de pájaros (Wong, 1983: 13). Es, junto con Héctor Eduardo Paniagua, sostiene Jesús Morales Bermúdez (1997: 63), el autor consagrado de esa compilación.

Armando Laparra, poeta guatemalteco, se refirió a la poesía de Serrano en una charla, sustentada en la Escuela Normal y Preparatoria de Tuxtla Gutiérrez, atractiva por el conocimiento de la poesía mexicana desplegado por Laparra y por la ubicación que hace del poeta chiapaneco: con huellas del romanticismo, se acercó al parnasianismo y al simbolismo, cuya muestra es percibida por Laparra en el poema "Los cisnes". La de Serrano, precisa, es una poesía que ofrece las dudas de un alma bohemia, cuya mirada no se aparta de la claridad ofrecida por el hogar cercano, desde el que cantó la condición del indio. No obstante, para Laparra el poeta aún estaba por llegar, aún no había aparecido el poeta Serrano que, sin imitaciones, cantara sus vivencias revolucionarias.

Las referencias anteriores invitan a adentrarse en la obra de Santiago Serrano, pero tal recorrido parece estar condicionado por las imprecisiones existentes respecto a sus libros, los cuales fueron publicados por él mismo en la imprenta La Sirena, en Tuxtla Gutiérrez. ${ }^{4}$ La definición de una historia literaria chiapaneca obliga a indagar en la obra de todos aquellos que han sido reconocidos como los formadores de tal literatura; una exploración que habrá de pasar, sobre todo, por la fijación de los textos. Ello impone el creador del poema motivo de este ensayo. Una clara distancia geográfica impide acometer dicha tarea. 
Ahora se intenta leer el poema antes citado a la luz de algunos de los que su autor propuso para su inclusión en Fiesta de pájaros. Si bien se ofrece una lectura con base en ciertos parámetros, se quiere enfatizar también el espacio donde ocurre dicho poema y la transformación que ahí sufre el sujeto de la enunciación. Ese espacio es la ciudad de New York. Interesa observar qué se tematiza en ese lugar. Una cuestión aparte es la veracidad de la fecha, 1917; sobre tal veracidad no se ahondará esta vez. No se está en condiciones, por el momento, de arremeter tal proyecto.

Todo parece indicar que quienes han aludido a este poema tomaron como ciertos lugar y fecha; no los han cuestionado, no han aportado datos que garanticen su veracidad. Llevar a cabo esa investigación será crucial para determinar los alcances del poema, no sólo por las palabras incluidas en él, sino también por el espacio donde se ubica y por las características de la mujer que ahí habita.

De ser ciertos ambos datos, en el poema estaría una de las primeras muestras de la utilización de anglicismos en la poesía escrita en América. ${ }^{5}$ Los préstamos lingüísticos anotados por Salomón de la Selva en "El soldado desconocido", de 1922 — año en el que habrían de aparecer los textos más representativos de la vanguardia: Ulises, “Tierra Baldía", "la Semana de Arte Moderno en Sao Paulo, la revista Proa y el estridentismo" (Flores, 2005: 21)—, serían posteriores a los del poema de Serrano.

La comparación se puede ir tornando más compleja si se busca la presencia de anglicismos en otras muestras poéticas. Por ejemplo, no habría que ignorar, en dicho ejercicio, los dos poemas que aparecen en la sección "En Nueva York" del libro Alsoly bajo la luna, dado a conocer en 1918, en los que José Juan Tablada muestra a la mujer norteamericana y sitúa el siglo veinte en la ciudad de Nueva York (Valdés, 1991: 15). Posterior a estos dos poemas es "Nocturno alterno", publicado en $L i$-po y otros poemas, un libro de 1920. Más que este poema, ¿'los dos de "En Nueva York" estarían como telón de fondo del de Santiago Serrano?
Por lo anterior, la importancia del poema de la rubia norteamericana para la historia literaria, no sólo de Chiapas, podría estar más allá de lo señalado por quienes lo han visto circunscrito al ámbito de la entidad federativa. Queda anotada esta advertencia, con el propósito de que tiempo después, quizás, se realice la investigación documental necesaria para la ubicación del poema en su justa dimensión.

Por otra parte, en "Yo Adoro a una Rubia....", se ubica al sujeto de la enunciación en una ciudad, en un espacio que no le pertenece. Fue Charles Baudelaire, es preciso recordarlo, quien transformó la ciudad, París, en figura principal de sus poemas. Con él, la ciudad empieza a exhibirse con sus rostros demudados; hay en ésta los pasos de quien intenta extraerle sus misterios: "Una cosa que distingue radicalmente a Baudelaire de sus precursores románticos y de sus sucesores simbolistas y del siglo xx, es la forma en que lo que sueña está inspirado por lo que ve" (Berman, 2001: 140). Están en sus poemas los sótanos, los cafés, las calles de París: "yo paseaba tensando como un héroe los nervios" (Baudelaire, 1984: 124); los viejos, sin eufemismos, y los ciegos, por igual, sin eufemismos; la belleza fugitiva, la demasiado alegre, las mujeres condenadas: Lesbos; el dulce caminar de la noche. No se trataba sólo de nombrar los lugares o los seres descubiertos en la calle, sino de la voluntad de Baudelaire de "luchar hasta agotar sus energías con las complejidades y contradicciones de la vida moderna, de encontrarse y crearse en medio de la angustia y la belleza de su caos en movimiento" (Berman, 2001:171).

En América existe una relación estrecha entre las transformaciones a las que se vieron sometidas algunas de sus ciudades y el modernismo, según lo ha analizado Álvaro Salvador Jofre (2002) a partir de la obra de Rubén Darío, Julián del Casal, José Asunción Silva, Manuel Gutiérrez Nájera, entre otros. Son autores que vivieron su esplendor en los últimos veinticinco años del siglo xIX, y cuyo empeño creativo 
se plantó frente al crecimiento que en ese período experimentaron las ciudades hispanoamericanas en las que les tocó vivir.

Se trata de una vida moderna expresada así por Federico García Lorca en un libro, cuyo primer verso es el siguiente: "Asesinado por el cielo", escrito al estar viviendo en New York a fines de la década de los años veinte del siglo pasado: "La aurora de Nueva York gime/ por las inmensas escaleras/ buscando entre las aristas/ nardos de angustia dibujada" (Lorca, 1986: 121). Un libro en el que está "la balada de la gran guerra", en el que ante el "paisaje con dos tumbas y un perro asirio", "el caballo tenía un ojo en el cuello". Un libro en el que se lee: "No es el infierno, es la calle./ No es la muerte, es la tienda de frutas" (Lorca, 1986: 133). Y la ciudad es ésta: "Nueva York de cieno,/ Nueva York de alambres y de muerte" (Lorca, 1986: 137).

\section{II}

Ocho de los nueve poemas de Santiago Serrano incluidos en Fiesta depájaros incorporan lugar y fecha en donde, se supone, fueron escritos, excepto el último. El primero está fechado en Guatemala en 1914; el segundo, en San Cristóbal de Las Casas, Chiapas, en 1928; el tercero, en Villa de Suchiapa, en noviembre de 1927; el cuarto, en La Habana, en 1918; el quinto, en Comitán de Domínguez, en 1932; el sexto, en Tuxtla Gutiérrez, en 1919; el séptimo, en Comitán de Domínguez, el 28 de agosto de 1933; y el octavo, que rige este texto, en New York, en 1917.

Como puede observarse, los poemas no son presentados mediante una ordenación cronológica. Al primero, de 1914, le sigue uno de 1928, por ejemplo. Y los lugares indicados parecen determinar la temática de cada poema. El de Suchiapa, sólo para mencionar un caso, se titula "Todos Santos". El sujeto de la enunciación está, en una noche de Todos Santos, buscando la tumba de su madre, y ante tal dolor por el ser perdido, quien se encuentra a salvo, sin abrojos, se revela una verdad: "ya estoy muerto". El que está fechado en Comitán, el 28 de agosto, se titula "Chiapas", y en él se canta a la entidad en la fecha de su independencia.

Estas indicaciones, paratextos ${ }^{6}$ que brindan señales sobre los poemas, no son una constante en el resto de las muestras poéticas de dicho libro. Al observarlas en los de Serrano, pueden permitir una lectura de los que tematizan la relación amorosa, por igual, impulsan una pregunta sobre la presencia de dichos lugares en tales textos. Además, al aparecer la mención de una ciudad estadounidense en un poema que, se aventura, fue escrito cuando Serrano tenía veintiún años de edad, brotan, de inmediato, dos preguntas: ¿existe la información suficiente que haga prever la presencia del poeta en dicha ciudad? ¿Es ésta un paisaje cultural, a la manera de Rubén Darío? El poema ha sido colocado en un sitio preponderante dentro de la poesía definida como chiapaneca. En su cronología literaria-cultural, Wong anota, sin la referencia bibliohemerográfica, lo siguiente respecto al año 1917: "Santiago Serrano publica su poema Mi amazona, que rompe —según Casahonda Castillo- con los modelos literarios establecidos por el autor de Pinceladas"7 (Wong, 1983: 11).

El carácter errante de Santiago Serrano se deja ver en la nota de presentación escrita por Tomás Martínez, otro de los poetas de esta Fiesta de pájaros, quien dice que Serrano anduvo por Cuba, Centroamérica y Sudamérica, "en devota romería artística" (Paniagua, 1932: 281). Cuando estudiaba la preparatoria en Guatemala, cuenta Enoch Cancino Casahonda (2004), fue, al parecer, a Nicaragua en busca de Rubén Darío. Viajó, pero también se quedaba largas horas en la hamaca de su casa, la que, en ciertas ocasiones, cuando permanecían abiertas las dos hojas de abajo, de la puerta de cuatro, mostraba no al padre, sino a la hija, una escena que a Eraclio Zepeda le gusta recrear: el escándalo del sol obligaba a aguzar la vista para extraer 
de la penumbra de la casa el cuerpo que se extendía con toda libertad.

La fecha tan temprana del de la rubia norteamericana impulsa a refrenar cualquier lectura literal de ese paratexto. ¿Cómo comprender la serenidad del sujeto de la enunciación si quien escribió el poema tenía, en ese entonces, veintiún años? Como se ha advertido líneas atrás, mientras no se realice un concienzudo trabajo de investigación literaria no se estará en condiciones de tomarla como cierta. Mientas tanto, en este ensayo, se procura leer lo tematizado en el poema, y no tomar éste como constatación de las andanzas del poeta. Interesa indagar sobre lo construido en el poema a partir de la ciudad. Para observar lo anterior, se seguirán cuatro poemas en virtud de la temática que en éstos se desarrolla, sin perder de vista que el propósito de este texto es arribar al que se asienta en un espacio de la ciudad de New York.

\section{III}

En cuatro de los nueve poemas de Serrano, incluidos en Fiesta depájaros, se tematiza la relación amorosa. Pero resulta atractivo ver cómo se produce un movimiento en esa tematización que desemboca en el poema número ocho, el de la rubia norteamericana.

El primero, fechado en Guatemala, se titula "Un mendrugo de amor"; se trata de un soneto en el que hay, desde los primeros versos, un tono romántico, $\mathrm{y}$ una voz femenina que ante la solicitud del enamorado sólo responde: "Pasa otro día'/ porque he cerrado mi corazón". En los tercetos, se produce un cambio no provocado por el poeta, sino por la ironía que, a veces, se encierra en los propios acontecimientos: ella, después de una decepción amorosa, interroga a quien había rechazado en un principio. Y él responde con las mismas palabras de ella, mas no por venganza: “"pasa después, ven otro día',/ pues como tú....... cerré mi corazón!".
El segundo poema es el que está fechado en La Habana, el cual, pudiera pensarse, podría albergar cierto aroma tropical, cierta sonoridad de las palabras de esa ciudad caribeña. Pero no hay tal: el sujeto de la enunciación espera la llegada de la amada para iluminar la oscura fortuna de su sino; ambiciona que los ojos de ella disuelvan en la vida de él su "fulgor opalino", una vida impuesta a las rudas andanzas. Y tiene la certeza de que el encuentro no se consumará en virtud de su procedencia en la que su vida ha sido "pobre, melancólica y bruna". Después de esta definición, los cuartetos presentan lo siguiente:

¡Qué feliz, si en mi vida, una vez, sólo una diluyeran sus ojos su fulgor opalino! ¡Qué ventura, si al menos no sufriera ninguna de estas rudas andanzas que me impuso el Destino, y pudiera esperarla en mitad del camino para amarla en silencio a la luz de la luna!

El poema, sin abandonar el tono romántico del anterior, está regido por un "si pudiera". El sujeto lírico no podrá amarla a ella en silencio, a la luz de la luna. La ciudad no se entera de los sinsabores de él, quien, al anhelar una cita con la persona amada, constata la oscuridad con la que se ha definido su vida. Ni la ciudad le brinda la certeza de que dicha cita se realice. Pesa más lo que él trae consigo y no lo que la ciudad pudiera ofrecerle y con lo que destruiría esa pobre vida; la marca paratextual enfatiza el desamparo en el que está el sujeto de la enunciación: lejos de su tierra y con toda su vida a cuestas.

El tercero, el cual no es un soneto como los dos anteriores, retoma el peregrinaje del sujeto lírico, referido antes, quien ha transitado por otros territorios y vuelve con sed y con un dolor encima, y en una tarde detiene su paso ante el huerto de la amada. Está fechado en Tuxtla Gutiérrez, en 1919. Principia con la conjunción copulativa "y"; de inmediato, se instala la idea respecto 
a un diálogo que continúa aquí, en el poema. Y así prosigue, en las tres estrofas restantes, con la conjunción "y" colocada al principio de cada una de éstas.

El sujeto de la enunciación le habla a ella, quien ha saciado la sed de este peregrino que en una tarde, presa del calor, se detiene ante "el Huerto Sagrado de tu Reino interior". Le dice, en tiempo pasado, lo que ella misma le provocó; le explica las razones por las cuales se quedó en ese huerto, observado por el sujeto lírico, donde la vida de ella florece: "rosa, fuente, jilguero", y las tres entidades mencionadas proporcionan: "trino, gala, frescor". Esa vida se dice, pues, en un alejandrino, en el que se percibe las habilidades del poeta: "rosa, fuente, jilguero: trino, gala, frescor". ${ }^{8}$ La estrofa está así:

Y ví que era tu vida todo un florecimiento: rosa, fuente, jilguero: trino, gala, frescor, y tuve una dorada ilusión, la de un cuento oriental, en que hay una Princesa de amor.

Si el poema anterior fue el lugar donde la cita con la amada no se consumó, en este tercero está la tarde donde el sujeto lírico, a pesar de la sed y el dolor, vio el huerto en el cual mitigó sus pesares. La interioridad de ella es un huerto donde reina lo sagrado; es un escenario oriental, cuya construcción era un motivo frecuente dentro del modernismo. El sujeto de la enunciación ha vuelto de sus aventuras en las que el amor no se ha realizado: ¿debió viajar para constatar que éste se encontraba ahí, al alcance de su mano? Los paisajes por los que anduvo su vista no dejaron en él más que el pesar de ese amor que se escapaba. ¿Y al no dejarse imprimir las huellas de esos paisajes, al alejarse del mundo, no hay en él cierta actitud romántica: insatisfecho con el mundo, la gente y su tiempo?

Se lee en el poema la influencia que sobre Serrano ejerció Rubén Darío, como anota Enoch Cancino Casahonda (2004), sobre todo si se va hacia "Venus", un soneto que forma parte de $A$ zul. Pero no brota de él la contundencia erótica, y no sólo exótica, explotada por Darío con intensidad en su obra poética; sí está la intención de pintar con palabras ese huerto, cuya luminosidad aparece en un endecasilabo anotado líneas arriba. En Darío, hay que decirlo, existe un engolosinamiento por los paisajes que no son naturales, sino culturales (Salinas, 2005: 96), una exteriorización afincada entre los epígonos del poeta nicaragüense.

Es el florecimiento de ella el que ha dispuesto el jardín, y ahí no hay sombra de duda, no existe ninguna pugna que destruya su armónica disposición. Ese huerto marca el antes y el después del sujeto lírico, quien ansía calmar su sed, no en la fuente de sí mismo, y refugiarse en la frescura que se le ofrece. Así, con esa disposición, y en una ensoñación constante, el sujeto de la enunciación ha quedado atrapado en ese follaje, y no piensa en lo que vendrá después: en el polvo, en la sombra, en la nada. Ha quedado atrapado en la flor del instante, como se sugiere en el "Poema del otoño", de Rubén Darío. Es un sujeto lírico que con el cabello gris se ha acercado a los rosales del jardín - ¿qué tan gris puede ser el cabello si en 1919 el poeta contaba con veintitrés años de edad?-, no con la vitalidad de su modelo, pero sí dentro de la tradición anacreóntica: "la del amante que 'a pesar del tiempo terco' se aferra al placer y al goce, sin querer darse por enterado del curso de los días, y, en ellos, del correr de la felicidad amorosa a su fin" (Salinas, 2005: 131).

Después de haber descrito dicha tematización en tres de los cuatro poemas, puede resultar comprensible que éstos no estén ordenados de forma cronológica. Aunque no exige una mayor compenetración porque estuviera teñido de claroscuros, el tono, la actitud del sujeto de la enunciación y la de la mujer presentan el cuarto poema en un sitio aparte de los anteriores. No se observa ya el tono melancólico, y cierto espacio idílico, que había prevalecido en los otros. En éste se trata de una mujer que está dispuesta, de manera distinta, a albergar la sed del sujeto lírico, quien en los anteriores 
había permanecido a la espera de un amor que lo salvara de su sino. En el cuarto poema se produce un cambio que se verá, con cierto detalle, en las siguientes líneas.

En los dos primeros versos del cuarto poema, el sujeto de la enunciación expresa sobre quién ha depositado su adoración. En el resto del poema dice cómo llegó a tal estado. De esos dos primeros versos habría que mirar el tiempo verbal, expresado en presente de indicativo, el artículo indefinido y, por sobre todas las cosas, el tipo de mujer y la actividad a la que ella se dedica. Es un yo enfático que patentiza su veneración hacia una mujer con características precisas: rubia norteamericana que juega tenis y baila fox-trot, $y$ sus rudas manos y sus ojos verdes lo sugestionaron.

La débil voluntad de él ha sido atrapada por esa rara mezcla de ella, y no otra podía ser la palabra para decir cómo él ha sucumbido ante quien, sin rodeos, lo conmina a abandonar a esa musa llorona que lo ha mantenido entre sueños, humo y mentira. Ella en un tiempo corto lo transformó en alguien que mediante vida esforzada ha descubierto el triunfo, y no vive más sumido en el sentimentalismo. Ella, con carácter, "con risas locas y muecas paganas", ha llevado a él del idealismo hacia el materialismo. Y el poema es un canto a ese cambio vivido por el sujeto de la enunciación.

Ha quedado atrás el huerto aquel donde el sujeto lírico pudo saciar su sed. Y no se deja cautivar por una mujer que le han presentado en algún salón, y que atrapa a todos con su risa, como una divina Eulalia que ríe, ríe y ríe. Las risas de la rubia norteamericana, quien se mueve entre el juego y el baile, son locas y están orientadas hacia la transformación del sujeto de la enunciación; están dirigidas a él, y no a un destinatario impreciso.

Si bien la ciudad es el lugar del encuentro del poeta con la amada, el espacio se cierra con la alusión a la actividad de ella y al sitio donde se la presentan: la cancha de tenis. En un breve repaso por los escenarios en los que ha sido dibujada la amada, destacan los salones pintados por Darío, el desierto de Othón y las calles por las que va la duquesa Job, cuya figura se muestra en "el primer poema que se escribe para una clase media urbana" (Pacheco, 1999: XL). Las mujeres de López Velarde transitan, con nombre propio, entre el corredor de la casa, llevadas por un luctuoso ruido de lino — una de ellas llevaba en el rostro "la embriaguez como un relámpago"- y las visitas engañosas a un parque de la ciudad que no les pertenece. Mujeres en un vaivén como la pequeña pelota que va entre una y otra raqueta, y ésta, en el poema de Serrano, en manos, que han perdido toda delicadeza, pertenecientes a una mujer semejante a las dibujadas por José Juan Tablada en el primer poema de la sección "En Nueva York", del libro Alsoly bajo la luna:

Mujeres 'fire proof', a la pasión inertes, llenas de fortaleza, como las cajas fuertes (Tablada, 1991:327).

Es la rubia una mujer que no está tras una ventana; se halla sumida en su propio universo, en movimiento ambos: mujer y universo. Es quien toma la iniciativa, y no está esperando a que el poeta, por fin, se decida a resolver sus propias contradicciones para declararle a ella sus propósitos. No aplaza nada, pues en ella está la inquietud, y con ánimo varonil lo llama a él a que abandone el humo y la mentira. No hay indiferencia ni apatía como sí se observa en los versos de Tablada escritos líneas atrás.

Es una mujer cuyas características no aparecen en ningún otro poema de Fiesta de pájaros, en la que las mujeres confían en que el amado les declarará su amor; son pasivas, están aguardando ser colmadas de besos, besos que en el exceso habrán de provocar que el amado olvide sus penas, como se lee en "Tus besos", de Galileo Cruz Robles, o en "Te quise por buena”, de Héctor Eduardo Paniagua. Las de dicha compilación son mujeres que bien pueden sintetizarse en estos versos 
de J. Antonio Rivera: “¿Eres risueña, espiritual y alada/ como un sueño de gloria y de ventura!" (Paniagua, 1932:276). Y el poeta se deja atrapar por los ojos de quien luce risueña, espiritual y alada.

Con esa mujer, Serrano pone los ojos en lo nuevo, y deja todo ese pasado en el que no había más que sentimentalismo. Se sumerge en el ahora, y no se lamenta por lo que ha quedado atrás, como habrían de hacerlo los románticos, quienes se lanzaron a conquistar paraísos que no encontraban ningún asidero en la realidad. Sus vueltas por el mundo, quizás, le habían mostrado que de la vida moderna podía surgir también la poesía. El tiempo fugitivo, irreparable, no le inquieta. No se fuga de un presente; se reafirma en éste. En las manos rudas de dicha mujer está sintetizada esa rara belleza que no había tenido cabida en los poetas anteriores a Serrano, quienes habían dibujado mujeres frágiles sin ningún parecido con esta amazona.

Ella es habitante de la ciudad, un espacio que había sido visto como sinónimo de destrucción, en el que no había más que inseguridad, y los pasos del transeúnte no podían detenerse, pues eran conducidos por el vértigo que ese espacio imprimía a todos los que ahí habitaban, como se ha ejemplificado al final del apartado uno de este texto. Errante como Caín, sin tierra promisoria, el transeúnte había de fatigarse por calles y calles, sin saber bien a bien lo que buscaba, sin encontrar más que la inquietud de su propia existencia: el desencanto de la vida moderna. Pero en la ciudad de Serrano es posible la vida.

Una idea más que se aleja de lo que se había escrito con anterioridad, de lo que estaban creando los contemporáneos de Santiago Serrano, es la relacionada con la vida esforzada, de la que sólo se puede obtener, apuntó Fray Luis, un ceño severo, pero en la que el sujeto de la enunciación de Serrano se halla tan a gusto. No sólo ha caído en manos de la rubia norteamericana sino que ha consentido vivir en el sitio al que ella lo ha llevado, en el que todo es producto del esfuerzo individual, y no lo que obtiene un grupo de hombres al enfrentarse con la naturaleza, como se mira en "Los trabajadores del bosque”, de Rodulfo Figueroa:

$\mathrm{Al}$ pie de aquellos árboles copudos Como negros fantasmas se agitaban Los atletas desnudos Que ardorosos se erguían o encorvaban, Mientras que, presas en sus puños rudos, Las hachas, cual relámpagos, brillaban (Figueroa, 1999:223).

Se ha comentado cómo la novedad de Serrano se puede extraer, por igual, de la disposición de los poemas en la selección de Fiesta de pájaros. Un signo de modernidad fue la conciencia con la que el poeta concibió su obra, en ello puso el énfasis Edgar Allan Poe, y luego Baudelaire, por ejemplo; en ello recala Pedro Salinas (2005) al reconstruir la complejidad con la que aparece lo erótico en Rubén Darío. Serrano no colocó sus textos de acuerdo con el tiempo en que fueron escritos. Si hubiera sido así, el de la rubia norteamericana habría sido el segundo. Pero al dejarlo en penúltimo lugar, dio muestras de esa conciencia con la que una obra moderna cobra forma. ${ }^{9}$ No acumuló los poemas, sino que los leyó y propuso una manera en que un lector futuro podría enfrentarse a ellos, un ejercicio que se ha aventurado ahora. ${ }^{10}$

\section{IV}

Santiago Serrano trasladó su sujeto lírico hacia la ciudad y lo convirtió en alguien que cayó rendido por el ímpetu de la rubia americana y del esfuerzo impreso en la vida que ella le propuso. Se arriesgó a construir un tipo de mujer, se inclinó por un modo de vida y dejó una mirada condescendiente sobre la ciudad. Sin embargo, a pesar de la inclusión de dos anglicismos, de los encabalgamientos, no logró abandonar los versos rimados; quedó atado a un ritmo que le impuso una rima que no supo seguir los 
vaivenes de la raqueta empuñada por su amada. Dirigió su mirada hacia la ruda mano de ella, pero no pudo resistir la fuerza de los ojos que lo sugestionaron. Aún en él, los ojos seguían siendo el espacio por donde podía llegarse a la interioridad de la amada.

El descubrimiento de la ciudad permitió que los escenarios fueran otros, que lo nombrado surgiera de lo que se extraía de ella, como la comparación hecha por Rodulfo Figueroa: "Que la vida es producto/ De la sangre y de los nervios, / Como la chispa que brota/ Del oculto motor eléctrico". O esta otra que anota versos más abajo, en sus rimas: "Que todos los hombres somos/ Máquinas de carne y hueso” (Figueroa, 1999: 78). A ello llegó Figueroa, porque lo que hay en él es el pueblo, en la falda de la montaña, con su cruz en la entrada, con su ermita, con sus dos cocoteros olvidados, con la alcaldía: un lugar por el que no ha pasado el tiempo. Considera también la hacienda, "con honores de pueblo y de castillo,/ Que da pingües cosechas por ofrenda/ A su viejo señor de horca y cuchillo" (Figueroa, 1999: 105). Y Tuxtla, para 1893, un año después de haberse convertido en la capital de Chiapas, es el pueblo de sus amores.

La ciudad, no una ciudad de Chiapas, habría de empezar a nombrarse, en la poesía reconocida como chiapaneca, con Santiago Serrano, como se ha visto en este texto, con todos los matices aquí señalados. Pero, al parecer, no hubo más en él que lo llevara a una exploración de ese mundo que se atrevió a tocar. Es posible advertir que prefirió dejar en sus poemas la quietud que le transmitía "la paz provinciana de la finca 'La Soledad"', como observa Tomás Martínez (Paniagua, 1932: 282), en la nota de presentación de los poemas de Serrano incluidos en Fiesta de pájaros. Había que esperar a que la ciudad se desposara con la noche. A que, con Jaime Sabines, llegaran, con "imposibles imágenes / y una gran libertad desconocida", miss x y la alegría de las azoteas con su ropa volando: ¡Qué alegre el ruido amontonado de la calle/ y el susto del rayo que cayó ahí cerca/ y los cláxones trepados uno encima del otro/ y la lluvia arreciando, apagándome el radio,/ mojándome los pulmones, cerrando las ventanas" (1991: 85). Había que esperar a que apareciera la ciudad bajo el relámpago, la de Efraín Bartolomé, a que la ciudad estuviera contra el cielo: "Los guardias de noche/ no saben si duerme./ Los guardias de día/ la pierden entre esbeltas columnas" (Macías, 1993: 33); a que el yo del poema expresara: "Yo no habito ciudad" (Vásquez Aguilar, 1991:18).

\section{Notas}

${ }^{1} \mathrm{El}$ autor agradece los comentarios de quienes leyeron alguna de las versiones de este texto: Guadalupe Olalde, Astrid Pinto Durán, Jesús Morales Bermúdez, Luis Arturo Guichard, Martín de la Cruz López y Pablo Salmerón.

${ }^{2}$ Santiago Serrano lo incluyó en su libro Del torbellino de mi vida (1940) con el título "Mi amazona", como habrá de ser nombrado también en un libro posterior de 1949. En el presente escrito se ha dejado el que aparece en el libro impulsado por Héctor Eduardo Paniagua, además, se ha respetado la disposición tipográfica, la puntuación y las palabras de esa edición (1932: 290-291).

${ }^{3}$ Óscar Wong presenta el poema con variantes en la puntuación y en una palabra, en lugar de "sueño", anota "ensueño".

${ }^{4}$ Según se lee en Aproximaciones a la poesía y la narrativa de Chiapas, de Jesús Morales Bermúdez, los libros de poesía de Santiago Serrano son los siguientes: La canción del grumete (1924), Del torbellino de mi vida (1940), Hojarasca, Plañideras, Las palomas de la tarde. Del momento humorístico (s/f), Playa a la vista (1949) y Sofreno mi caballo (s/f).

${ }^{5}$ Como lo ha hecho ver, mediante comunicación electrónica, el doctor Luis Arturo Guichard.

${ }^{6}$ Un paratexto es un "texto que es un campo de relaciones, un lugar privilegiado de la dimensión pragmática de la obra por su relación con el lector, ya que aporta señales accesorias que procuran un entorno al otro texto. Tales son, respecto de un libro, sus epígrafes, títulos, subtítulos, intertítulos, prefacios, advertencias, prólogos, esquemas previos, proyectos, borradores, notas, epilogos, solapas, etc.”(Beristáin, 2000:271). Para el caso que aquí se trata, el paratexto está dado por la indicación del lugar y la fecha de escritura de cada uno de los poemas. También lo es la ficha de presentación de cada uno de los poetas que forman Fiesta de pájaros. 
${ }^{7}$ El autor de Pinceladas es Rodulfo Figueroa, identificado como el iniciador de la poesía moderna en Chiapas.

${ }^{8}$ Sobresale, por igual, el hecho de que las entidades de ese florecimiento estén dichas en singular; no se trata de las rosas, sino de la rosa; no de los jilgueros, sino del jilguero. Sólo se menciona que la tematización del huerto, del jardín, fue una constante en la poesía bucólica. No obstante, es posible observar cómo el jardín ha sido tematizado por algunos poetas, por ejemplo, en el ensayo que Elsa Cross escribió a partir de la poesía de Octavio Paz, titulado "Los dos jardines".

9 El último es un soneto, titulado "Sofreno mi caballo...", cuyo primer verso va así: "Sofreno mi caballo bajo el frescor de un pino..." (Paniagua, 1932: 292).

${ }^{10}$ El ejercicio, en el que se ha corrido un riesgo, queda frente al abismo al leer la advertencia con la que es presentada la obra poética de Rodulfo Figueroa: "El ordenamiento temático de la obra poética de Rodulfo Figueroa fue realizado por Ricardo Cuéllar Valencia, investigador del presente volumen" (Figueroa, 1999: 70).

\section{Bibliografía}

Baudelaire, Charles, 1984, Las flores del mal, introducción, traducción en verso y notas de Carlos Pujol, Planeta, Clásicos Universales 89, Barcelona.

Beristáin, Helena, 2000, Diccionario de retórica y poética, Editorial Porrúa, octava edición, segunda reimpresión, México.

Berman, Marshall, 2001, Todo lo sólido se desvanece en el aire. La experiencia de la modernidad, decimotercera edición, Siglo XXI Eds., México.

Cancino Casahonda, Enoch, 2004, “Santiago Serrano, un recuerdo navideño", Cuarto Poder, 31 de diciembre, Tuxtla Gutiérrez.

Darío, Rubén, 1993, Prosas profanas, Espasa-Calpe, colección Austral 404, duodécima edición, México.

Figueroa, Rodulfo, 1999, Obrapoética, edición de Ricardo Cuéllar Valencia, Consejo Estatal para la Cultura y las Artes, Libros de Chiapas, Temas representativos, México.

Flores, Miguel Ángel, 2005, "Introducción”, El soldado desconocido y otros poemas, Salomón de la Selva, primera reimpresión de la primera edición, FCE, Colección Tierra Firme, México.

García Lorca, Federico, 1986, "Poeta en Nueva York", Libro de poemas, poema del cante jondo..., prólogo de Salvador Novo, Ed. Porrúa, colección Sepan Cuántos... 251, México.

Laparra, Armando, 1993, "Trayectoria lírica de Santiago Serrano", s/e, pp. 123-125.

Macías, Elva, 1997, Ciudad contra el cielo, CONACULTA, colección Luzazul, México.

Morales Bermúdez, Jesús, 1999, Aproximaciones a la poesía y la narrativa de Chiapas, Universidad de Ciencias y Artes de Chiapas, segunda edición, Tuxtla Gutiérrez.

Pacheco, José Emilio, 1932, Antología del modernismo (18841921), UNAM-Ediciones Era, Biblioteca del Estudiante Universitario 90-91, tomos I y II en un volumen, México.

Paniagua, Héctor Eduardo, 1991, Fiesta de pájaros, Imprenta del gobierno del estado, Tuxtla Gutiérrez.

Sabines, Jaime, 1991, Otro recuento de poemas 1950-1991, Joaquín Mortiz-Instituto Chiapaneca de Cultura, México.

Salinas, Pedro, 2005, La poesía de Rubén Dario, Ediciones Península, colección historia, ciencia y sociedad número 342, Barcelona.

Salvador Jofre, Álvaro, 2002, El impuro amor de las ciudades (Notas acerca de la literatura modernista y el espacio urbano), Casa de las Américas, La Habana.

Tablada, José Juan, 1991, Obras I. Poesía, recopilación, edición, prólogo y notas de Héctor Valdés, UNAM, Instituto de Investigaciones Filológicas, Nueva Biblioteca Mexicana 24, México.

Valdés, Héctor, 1991, "Prólogo", Obras I. Poesía, José Juan Tablada, UNAM, Instituto de Investigaciones Filológicas, Nueva Biblioteca Mexicana 24, México.

Vásquez Aguilar, Joaquín, 1991, Erguido a penas, Gobierno del estado de Chiapas-Instituto Chiapaneco de Cultura, Tuxtla Gutiérrez.

Wong, Óscar, 1983, Nueva poesía de Chiapas (antología), Ed. Katún, México.

—, 1998, Chiapas. Nueva fiesta de pájaros, Ed. Praxis, México. 